\title{
Protein intake and bone health across the lifecycle: an updated systematic review and meta-analysis of the evidence
}

\author{
D. Wynter ${ }^{1}$, R. Manders ${ }^{1}$, D.J. Torgerson ${ }^{2}$, C.E. Hewitt ${ }^{2}$, D.J. Millward ${ }^{1}$, S.A. Lanham-New ${ }^{1}$ \\ and A.L. Darling ${ }^{1}$ \\ ${ }^{1}$ Department of Nutritional Sciences, School of Biosciences and Medicine, Faculty of Health and Medical Sciences, \\ University of Surrey, Guildford and \\ ${ }^{2}$ Clinical Trials Unit, University of York, York
}

The relationship between dietary protein intake and bone health has sparked intense debate for many years, and there is still considerable controversy as to whether protein is an important nutrient for optimising skeletal integrity during the life-cycle. The aim of this study was to undertake an updated systematic review and meta-analysis to fully examine the relationship between dietary protein and bone health in the healthy human adult population following the 2009 protein and bone health meta-analysis publication by Darling et al. $(2009)^{(1)}$.

The PUBMED (September 2007 to January 2016) database was electronically searched for relevant studies of healthy adults. A total of 1913 potentially relevant studies using the PUBMED database and 3 hand-searched studies were found. Results of the cohort and cross-sectional surveys; all of the pooled correlation coefficients were positive but mostly not statistically significant $(\mathrm{P}>0 \cdot 05)$. Hence, the proportion of the variance in bone mineral density BMD) explained by protein intake is unclear (random-effects model used due to high heterogeneity).

A meta-analysis of randomised, placebo-controlled trials was conducted and this suggested no statistically significant positive effect of whey protein supplementation on BMD at the femoral neck (random-effects model, $\mathrm{P}=0 \cdot 23$ ) or the total hip (fixed-effects model, $\mathrm{P}=0.56$ ). However, a statistically significant effect of whey protein supplementation on femoral neck BMD was found using the fixed-effects model (Mean Difference $=-2.03[-2.44,-1.63] \mathrm{P}<0.001$ ). Nonetheless, the random-effects model was more appropriate due to the considerable heterogeneity found $\left(\mathrm{I}^{2}=82 \%, \mathrm{P}=0.02\right)$. It was not possible to run meta-analyses on total protein, soy protein, animal protein or vegetable protein due to low quality studies, cross-over supplementation study designs used or incompatibility with other studies.

Evidence suggesting a deleterious effect of dietary protein on bone health was not found in this analysis - indeed the results demonstrated a positive effect of protein in bone at different sites but no effect of whey protein supplementation on BMD at the femoral neck or total hip. Overall, the strength of evidence suggests a weak beneficial effect of dietary protein on bone health.

Further analysis of the available literature is now underway to combine this new (2007-2016) data with respect to our previously published meta-analysis (pre-2007 publications). However, according to the most recent National Diet and Nutrition Survey (NDNS), the UK population surpasses the reference nutrient intake (RNI) for protein and thus, a protein intake increment seems unlikely to provide a further benefit to bone health.

1. Darling AL, Millward DJ, Torgerson DJ et al. (2009) Am J Clin Nutr 6, 1674-1692. 